Background In addition to biomedical treatment, healthcare providers (HCPs) may make psychosocial contributions to injured workers that aide rehabilitation and the return to work (RTW) process. We examined the effect on disability duration of several types of HCP communications with injured workers and stakeholders in the RTW process.

Objectives To test the effect of various HCP communications on time off work following injury.

Research design We analysed survey and administrative claims data from $n=715$ injured workers in Victoria, Australia. Survey responses were collected around five months post-injury and provided data on HCP communication and confounders. Administrative claims data provided data on compensated time off work. We conducted multivariate zero-inflated Poisson regression analyses, which evaluated both the likelihood of future time off work and its duration.

Measures HCP communications included good interactions, estimated RTW date, activity discussions, prevention discussions, and stakeholder contact. Time off work was the count of cumulative compensated work absence in weeks, accrued post-survey.

Results Only RTW dates were predictive of no future time loss (OR: 2.65, 95\% CI: 1.74-4.03). RTW date (IRR: 0.71, 0.67-0.74), good interactions (IRR: 0.73, 0.70-0.76), and stakeholder contact (IRR: 0.92, 0.88-0.95) reduced time off work, while activity discussions predicted more time off work (IRR: 1.13, 1.08-1.19).

Conclusions HCPs may be able shorten disability durations through several types of communication. Of those evaluated in this study, RTW dates had the most robust effect.

\section{P.2.11 VITAMIN D DEFICIENCY AND HEPATITIS B VIRUS INFECTION AND RISK OF HEMATOLOGICAL MALIGNANCIES AMONG KOREAN SEMICONDUCTOR WORKERS: A CASE-CONTROL STUDY}

${ }^{1}$ Seokwon Lee, ${ }^{1}$ Sarah Yang, ${ }^{1}$ Jaewoo Koh, ${ }^{2}$ Soo-Geun Kim, ${ }^{1}$ Kyoungho Lee*. 'Samsung Electronics Co., Ltd., Hwaseong, Gyeonggi-do, Republic of Korea; ${ }^{2}$ Sungkyunkwan University, School of Medicine, Suwon, Gyeonggi-do, Republic of Korea

\subsection{6/OEM-2019-EPI.245}

Objectives This study aims to identify an association between potential risk factors and risk of hematological malignancies, especially for Non-Hodgkin's Lymphoma (NHL) and leukemia, among Korean semiconductor workers.

Methods We collected 54 cases who ever worked over 30 days at a Korean semiconductor manufacturing company and were diagnosed as NHL (ICD-10 codes: C82-C85) and leukemia (C91-C95) from January 1 st 1998 to December 31 st 2017. A total of 324 controls, frequency matched by age and sex (1:6 ratios) with no diagnosis of hematological malignancies, were randomly selected. The information on work history, occupational exposure and medical examination data was also collected for the study analysis. Multivariable logistic regression analyses were performed to estimate odds ratios (ORs) after adjusting age, sex, education, employment year, smoking, alcohol and body mass index.

Results No significant association between hematological malignancies and occupational risk factors, including job title, cleanroom work and occupational exposures, was observed. As for leukemia, however, ORs were significantly increased by natural immunity to hepatitis $\mathrm{B}$ virus (HBV) $[\mathrm{HBsAg}(-), \mathrm{HBsAb}(+), \mathrm{HBcAb}(+)] \quad(\mathrm{OR}=11.92,95 \%$ confidential interval 1.05-135.89) and a past or current (ever)
HBV infection [HBsAg(+/-), $\mathrm{HBsAb}(+/-), \mathrm{HBcAb}(+)]$ (6.52, 1.51-28.10). Furthermore, ORs for NHL were also significantly increased by insufficient serum 25 -hydroxyvitamin D [25(OH)D] (12-20 ng/mL) $(10.67,1.27-89.53)$ and deficient $25(\mathrm{OH}) \mathrm{D}$ levels $\quad(<12 \mathrm{ng} / \mathrm{mL}) \quad(12.22, \quad 1.37-109.16)$ $(\mathrm{p}<0.05)$.

Conclusions Risk of hematological malignancies was not associated with occupational risk factors but significantly elevated by two factors, such as HBV infection and insufficient or deficient 25(OH)D level. A longitudinal cohort study is needed to confirm the association between these risk factors and cancers.

\section{P.2.12 CLUSTERING OF MALIGNANT PLEURAL MESOTHELIOMA IN ASBESTOS FACTORIES IN A 29-YEARS FOLLOW-UP STUDY TO IDENTIFY HIGH-RISK INDUSTRIES IN TAIWAN}

$1,2,3,4$ Lukas Lee*, ${ }^{5}$ Cheng-Kuan Lin, ${ }^{6}$ Chih-Hong Pan, ${ }^{7}$ Yawen Cheng, ${ }^{1} Y u-Y i n$ Chang, ${ }^{1}$ Saou-Hsing Liou, ${ }^{8,9}$ Jung-Der Wang. 'National Institute of Environmental Health Sciences, National Health Research Institutes, Taipei, Taiwan; ${ }^{2}$ Department of Environmental and Occupational Medicine, National Taiwan University Hospital, Taipei, Taiwan; ${ }^{3}$ Institute of Occupational Medicine and Industrial Hygiene, College of Public Health, National Taiwan University, Taipei, Taiwan; ${ }^{4}$ Ph.D. Program in Environmental and Occupational Medicine, Kaohsiung Medical University., Kaohsiung, Taiwan; ${ }^{5}$ Department of Environmental Health, Harvard T.H. Chan School of Public Health, Boston, USA; ${ }^{6}$ Institute of Labor, Occupational Safety and Health, Ministry of Labor, New Taipei City, Taiwan; 'Institute of Health Policy and Management, College of Public Health, National Taiwan University, Taipei, Taiwan; ${ }^{8}$ Department of Public Health, College of Medicine, National Cheng Kung University, Tainan, Taiwan; ${ }^{9}$ Departments of Internal Medicine and Occupational and Environmental Medicine, National Cheng Kung University Hospital, Tainan, Taiwan

\subsection{6/OEM-2019-EPI.246}

Objective Exposure to asbestos is the major cause for malignant pleural mesothelioma (MPM) but the causal link of individual cases is difficult to establish for lack of exposure information and long disease latency.

Methods We established a retrospective cohort of workers employed in asbestos industries during the period of 19501989 and the occurrence of MPM during the period of 1980-2009 was examined with the Taiwan Cancer Registry. Estimated rate ratios (eRR) were computed for each factory where any case of MPM was diagnosed by assuming Poisson distribution with a minimal latency of 20 years.

Results A total of 18 MPM (17 males, 1 female) in 8 factories were found. The incidence rate of MPM for the 8 factories was 18.0 per million, ranging from 6.2 per million (military factory) to 268.2 per million (asbestos cement). We observed significantly increased risks for MPM in asbestos cement, thermal insulation and shipbuilding industries, with eRR (genders combined) of $113.6,87.5$, and 15.8 respectively. The sensitivity analyses considering latency showed similar findings in latency $\geq 30$ years, and the shipbuilding industry presented a significant $\mathrm{eRR}$ given a latency $\geq 40$ years. The gender-specific eRR showed similar results in men, but high eRR of 729.6 was observed in an asbestos cement factory where a female MPM was diagnosed.

Conclusions This nationwide study in Taiwan comprehensively showed different asbestos manufacturing processes, including asbestos cement, thermal insulation, and shipbuilding industries, at significantly increased risks for MPM. We recommend to establish a medical screening program for workers previously exposed to asbestos to identify MPM and other asbestos-related diseases at an earlier stage. 